\title{
Original
}

\section{Magnetic Resonance Imaging Signal Intensities of the Temporomandibular Joint Articular Disc and Cortical Bone : Are These Measurements Valid for the Diagnosis of Temporomandibular Joint Disease?}

\author{
Mamiko FuJIKURA $^{* 1)}$, Keiichi NishikawA ${ }^{2)}$ and Kazuyuki ArAKI ${ }^{3)}$
}

\begin{abstract}
The purpose of this study was to clarify the validity of measuring the signal intensities of cortical bone (CB) and temporomandibular joint (TMJ) discs on magnetic resonance (MR) images for the diagnosis of TMJ disease. Two investigations were performed. In the first investigation, MR images of 13 TMJs of volunteers were obtained using a multi spin-echo sequence. Echo time (TE) was increased from $10 \mathrm{msec}$ to $200 \mathrm{msec}$ in steps of $10 \mathrm{msec}$. The signal intensities of the $\mathrm{CB}$ of the condylar head, and the anterior (AD) and posterior (PD) bands of the TMJ disc were measured, and their attenuation patterns were compared. In the second investigation, clinical MR images of 30 TMJs from patients with suspected TMJ disorders were selected. Proton density-weighted images (PDWIs) and T2-weighted images (T2WIs) were obtained at TEs of 14 and $85 \mathrm{msec}$, respectively, using a double-echo fast spin-echo sequence. The signal intensities of $\mathrm{CB}, \mathrm{AD}$, and PD were measured and compared with that of the external auditory meatus (air) using a Bonferroni test. $\mathrm{AD}$ and $\mathrm{PD}$ showed nearly identical signal intensity attenuation patterns. The signal intensities of $\mathrm{AD}$ and $\mathrm{PD}$ were lower than $\mathrm{CB}$ at TEs longer than $20 \mathrm{msec}$ although they were higher at TEs shorter than $20 \mathrm{msec}$. Clinical PDWIs and T2WIs showed similar results. The signal intensities of CB, $\mathrm{AD}$, and $\mathrm{PD}$ were significantly higher than the external auditory meatus (air) in both PDWIs and T2WIs. $\mathrm{CB}$ and TMJ discs show characteristic signal intensities, suggesting that the signal intensity measurement of these structures is valid.
\end{abstract}

Key words : magnetic resonance imaging, temporomandibular joint disc, cortical bone, signal intensity

\section{Introduction}

Disorders of the temporomandibular joint (TMJ) comprise a group of diseases that involve symptoms in the area of the TMJ, such as pain or movement disorders. Temporomandibular disorders (TMDs) affecting the TMJ and/or jaw muscles are attributed to many causes. One cause of TMD is articular disc abnormality. The effectiveness of magnetic resonance imaging

\footnotetext{
1) Department of Oral Diagnostic Sciences, Division of Pathology, Showa University School of Dentistry, 1-5-8 Hatanodai, Shinagawa-ku, Tokyo 142-8555, Japan.

2) Department of Chemistry, Tokyo Dental College.

3) Department of Oral Diagnostic Sciences, Division of Radiology, Showa University School of Dentistry.

* To whom corresponding should be addressed.
} 


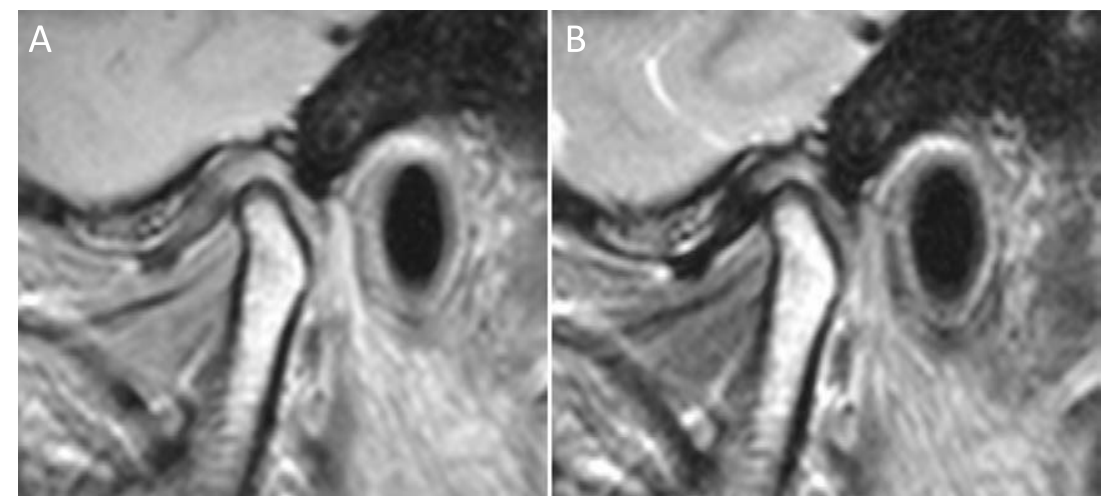

Fig. 1. Example magnetic resonance images of the temporomandibular joint. A) Proton density-weighted image; B) T2-weighted image. The anteriorly-displaced disc is evident in both images.

(MRI) in the diagnosis of TMD was first reported in the 1980 $\mathrm{s}^{1,2)}$, and MRI subsequently became a powerful diagnostic tool for $\mathrm{TMD}^{3,4)}$.

Fig. 1 presents example MR images of the TMJ. Proton density-weighted images (PDWIs) and T2-weighted images (T2WIs) are currently frequently used in clinical examinations. TMJ disc position and peri-articular inflammation are evaluated using these images.

There have been many MRI reports related to the position and shape of the TMJ disc, and its relation to clinical symptoms. Increased symptoms, which indicate a more advanced TMD, are found significantly more often with TMJ disc displacement without reduction, than in TMJ disc displacement with reduction on $\mathrm{MRI}^{5)}$. More severe disc deformity is seen in TMJ disc displacement without reduction, than in TMJ disc displacement with reduction ${ }^{6}$.

MRI is mainly used in the diagnosis of soft tissue lesions. Hard tissues, such as bone cortex and tooth enamel, show no signals on MRI, and signal intensity changes indicating disease in hard tissues are generally not observable on MRI. In the case of the condylar cortex, however, its form can be evaluated on MRI. Geiger et $a l^{7)}$ compared MRI and computed tomography for assessment of the condylar cortex using cadavers, and demonstrated the high accuracy of MRI measurements.

Few studies have evaluated the signal intensity of the TMJ disc and the cortex of the condylar head. We found only two reports on $\mathrm{T} 2$ relaxation time and $\mathrm{T} 2 *$ relaxation time. Kakimoto et $a l^{8)}$ compared the $\mathrm{T} 2$ relaxation time of the disc between patients with TMD and healthy volunteers, and showed that the T2 relaxation time in patients with TMD was longer than in healthy volunteers.

The purpose of this study was to clarify the validity of signal intensity measurements of the TMJ articular disc and condylar cortex. We measured their signal intensity in relation to echo time (TE), and then compared their signal intensity with that of air, in which the signal intensity is theoretically zero. 


\section{Materials and methods}

All experiments were approved by the ethic committee of the Tokyo Dental College (No.347 and No.521). The investigation was performed in two parts.

\section{Experiment 1: Comparison of signal intensity attenuation curves}

Nine volunteers (5 males, 4 females) participated in this part of the study. Their mean age was 28.8 years (range, 26-44 years). The volunteers had no symptoms in the TMJ region. MRI was performed using a 1.5 tesla unit (MAGNETOM Symphony; Siemens, Erlangen, Germany) with TMJ surface coils. Both TMJs were examined with a multi-echo sequence. Imaging parameters are shown in Table 1. TE was increased from $10 \mathrm{msec}$ to $200 \mathrm{msec}$ in steps of 10 msec. This imaging produced 20 images for each TMJ. Five of eighteen TMJs were excluded from the analysis because the anterior (AD) and/or posterior (PD) band of the TMJ disc was not observed clearly. On each image, circular regions of interest (ROIs) were placed at the

Table 1. Magnetic resonance imaging protocol for evaluating the relationship between signal intensity and echo time (TE)

\begin{tabular}{ll}
\hline TE & $10 \mathrm{msec} \sim 200 \mathrm{msec}$ \\
TR & 3,300 \\
ETL / NSA & $1 / 1$ \\
FOV & $150 \mathrm{msec} \times 150 \mathrm{msec}$ \\
Slice thickness & $3 \mathrm{~mm}$ \\
Slice gap & $0.3 \mathrm{~mm}$ \\
Matrix & $256 \times 256$ \\
\hline
\end{tabular}

TR, repetition time; ETL, echo train length; NSA, number of signals averaged; FOV, field of view.
Table 2. Magnetic resonance imaging protocol for evaluating the difference in signal intensity in patients with suspected temporomandibular disorders

\begin{tabular}{ll}
\hline TE & $14 \mathrm{msec}, 85 \mathrm{msec}$ \\
TR & 3,300 \\
ETL / NSA & $5 / 1$ \\
FOV & $150 \mathrm{msec} \times 150 \mathrm{msec}$ \\
Slice thickness & $3 \mathrm{~mm}$ \\
Slice gap & $0.3 \mathrm{~mm}$ \\
Matrix & $256 \times 256$ \\
\hline
\end{tabular}

TE, echo time; TR, repetition time; ETL, echo train length; NSA, number of signals averaged; FOV, field of view.

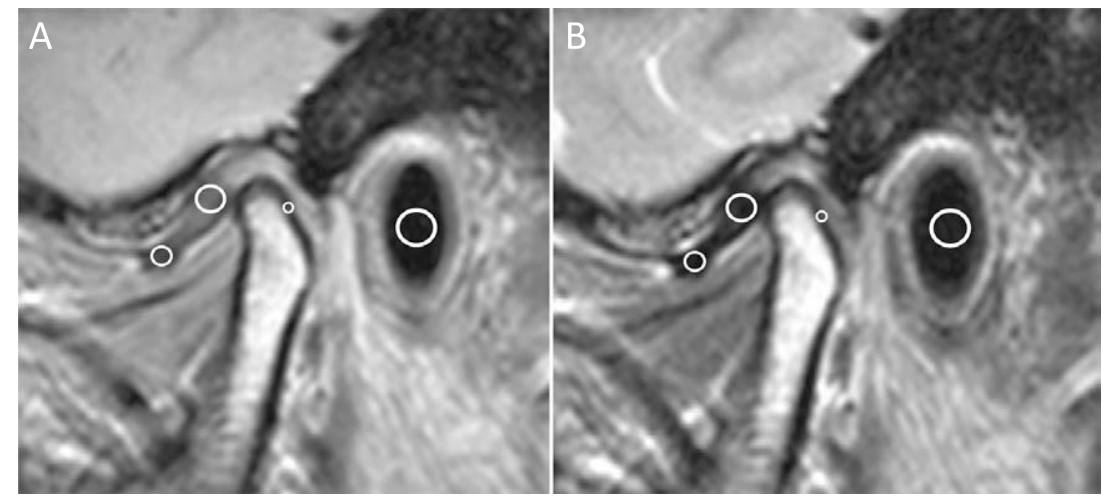

Fig. 2. Region of interest (ROI) settings for measurement of signal intensity. A) Proton density-weighted image ; B) T2-weighted image. ROIs were placed at the anterior and posterior band of the disc, the cortical bone of the condylar head, and the external auditory meatus (air). 
$\mathrm{AD}$ and PD of the TMJ disc, the cortical bone (CB) of the condylar head, and the external auditory meatus (air), as shown in Fig. 2. The signal intensity of each ROI was measured using the dedicated workstation of the MRI unit. The average signal intensity for each structure was calculated, and an attenuation curve of signal intensity was generated.

\section{Experiment 2: Comparison of signal intensity in clinical images}

Clinical MR images of TMJs were analyzed retrospectively. Fifty-six TMJ images from 28 patients ( 8 males, 20 females) were included in this part of the study. The mean age of these patients was 33.8 years (range, 14-71 years). The MR images were recorded at the Tokyo Dental College, Chiba Hospital, Japan, between September and October 2012. All patients complained of TMD. The MR images were obtained using a double-echo sequence, which produced PDWIs and T2WIs simultaneously. The imaging parameters are shown in Table 2. Twenty-six TMJs were excluded because the $\mathrm{AD}$ and/or PD of the TMJ disc could not be observed clearly. The remaining MR images of 30 TMJs were analyzed using the same method as in Experiment 1. Circular ROIs were placed at the AD, PD, CB, and external auditory meatus, as shown in Fig. 2. The signal intensity of each ROI was measured, and the average signal intensity for each structure was calculated. The Bonferroni test was performed for statistical analysis. The significance level was set at 5\% ( $\alpha=0.05$; EXCEL TOUKEI Ver. 6.06; Etsumi Co. Ltd., Tokyo, Japan).

\section{Results}

\section{Experiment 1: Comparison of signal intensity attenuation curves}

Example MR images acquired at the different TEs are shown in Fig. 3. Figures 4A, B, and $\mathrm{C}$ show the signal attenuation curves for each TMJ structure, corresponding to $\mathrm{AD}, \mathrm{PD}$, and $\mathrm{CB}$, respectively. The signal intensity at each TE showed bilateral and individual differences. The attenuation curves of $\mathrm{AD}$ and $\mathrm{PD}$ exhibited a similar tendency.

The attenuation curves generated with averaged signal intensities are shown in Fig. 5. Although the curves of $\mathrm{AD}$ and $\mathrm{PD}$ were almost identical, the curve of $\mathrm{CB}$ was quite different. At TEs shorter than $20 \mathrm{msec}$, the signal intensities of $\mathrm{AD}$ and $\mathrm{PB}$ were higher than $\mathrm{CB}$. At a
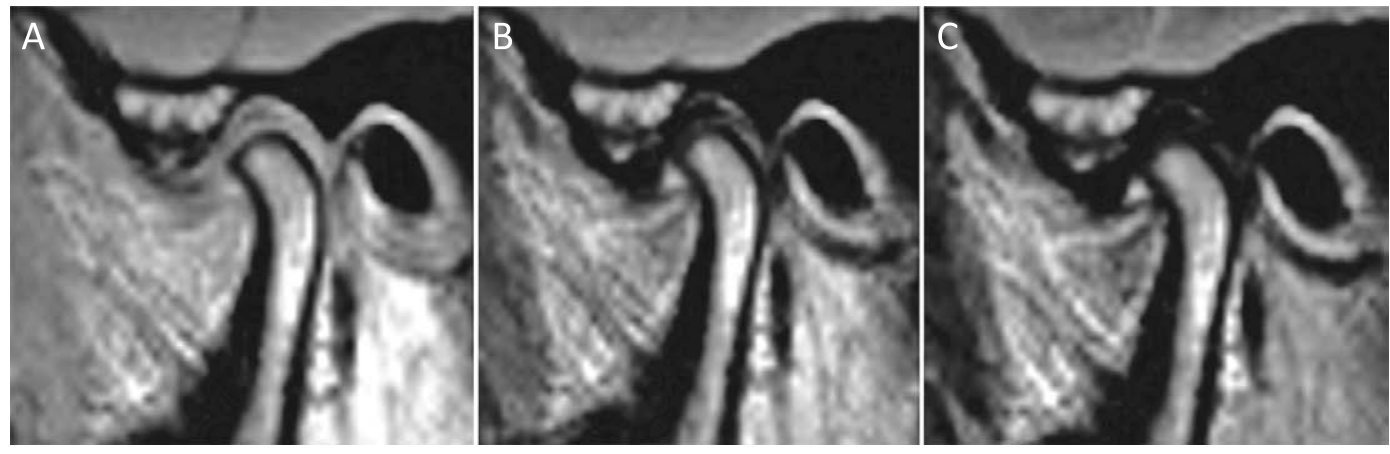

Fig. 3. Example magnetic resonance images showing changes in the signal intensity according to echo time (TE). A) $\mathrm{TE}=10 \mathrm{msec} ; \mathrm{B}) \mathrm{TE}=50 \mathrm{msec}$; C) $\mathrm{TE}=100 \mathrm{msec}$. Changes in the signal intensity of the disc are evident. 

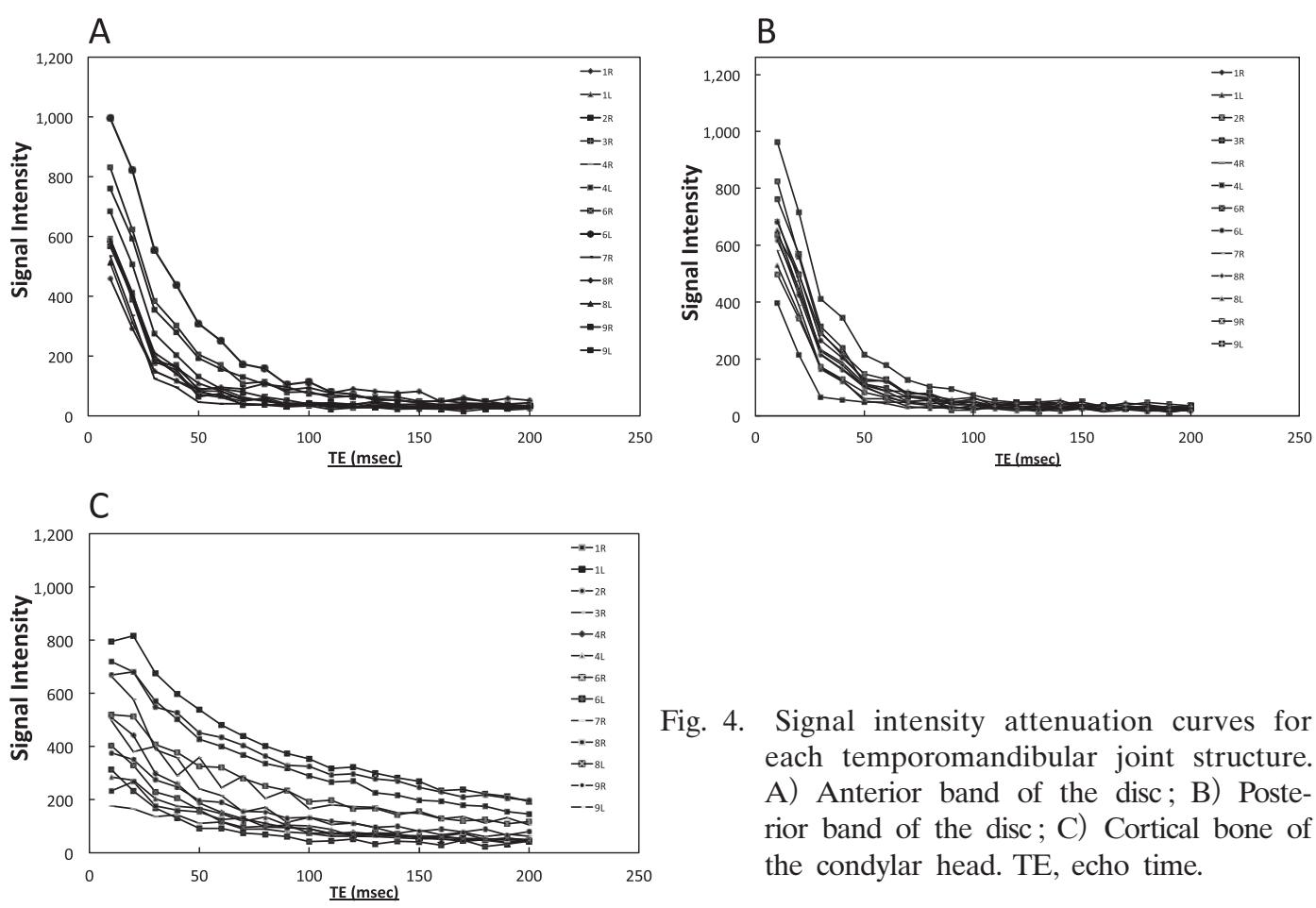

Fig. 4. Signal intensity attenuation curves for each temporomandibular joint structure. A) Anterior band of the disc; B) Posterior band of the disc; C) Cortical bone of the condylar head. TE, echo time.

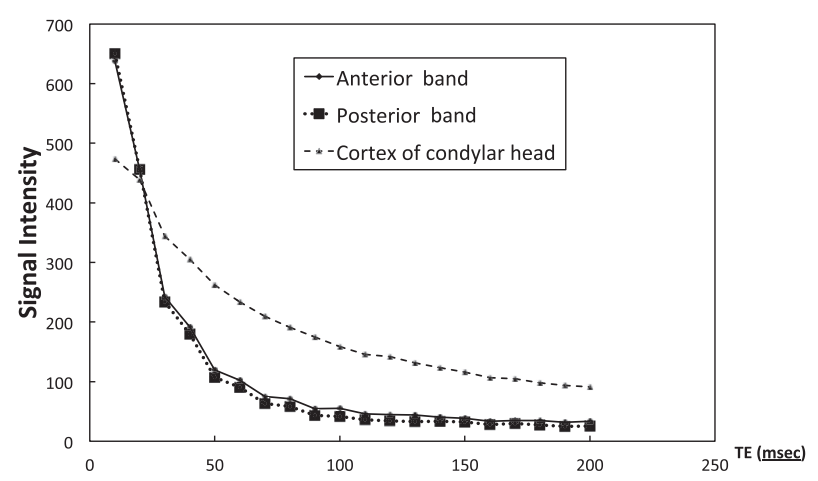

Fig. 5. Averaged signal intensity attenuation curves of the anterior band of the disc, posterior band of the disc, and cortical bone of the condylar head. TE, echo time.

TE of 20 msec, AD, PD, and $\mathrm{CB}$ showed almost the same signal intensity. At TEs longer than $20 \mathrm{msec}$, the signal intensity of $\mathrm{CB}$ was higher than $\mathrm{AD}$ and PD.

\section{Experiment 2: Comparison of signal intensity in clinical images}

The signal intensities of $\mathrm{AD}, \mathrm{PD}, \mathrm{CB}$, and the external auditory meatus are shown in Fig. 6. The signal intensities of $\mathrm{AD}, \mathrm{PD}$, and $\mathrm{CB}$ were significantly higher than those of the external auditory meatus (air) on both the PDWI and T2WI. AD and PD showed a higher signal intensity than $\mathrm{CB}$ on the PDWI; however, on the T2WI, $\mathrm{CB}$ showed a higher signal intensity than $\mathrm{AD}$ and PD. These results were similar to those obtained in Experiment 1. 

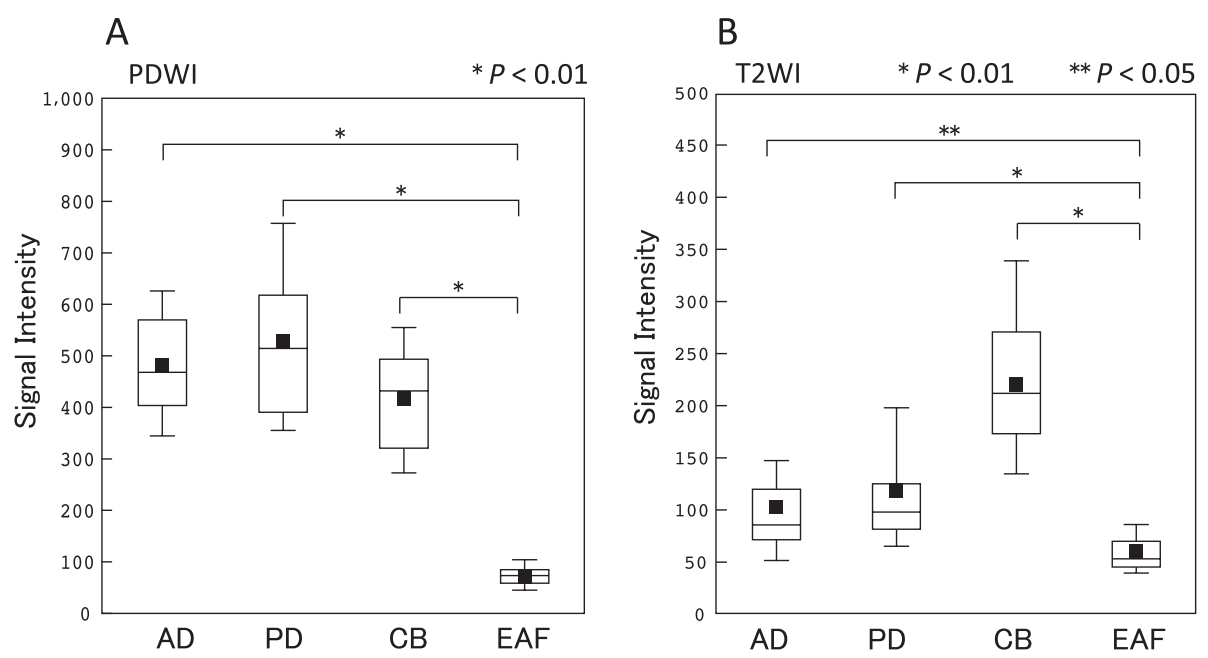

Fig. 6. Signal intensities of patients with suspected temporomandibular disorders. A : Signal intensity from proton density-weighted image (PDWI). B : Signal intensity from T2-weighted image (T2WI). Black squares represent the mean values. The horizontal line of each box represents the median, while the top and bottom borders of the box represent the $75^{\text {th }}$ and $25^{\text {th }}$ percentiles, respectively. Whiskers indicate maximum and minimum values. $\mathrm{AD}$, anterior band of the disc; $\mathrm{PD}$, posterior band of the disc; $\mathrm{CB}$, cortical bone of the condylar head; EAF, air of the external auditory meatus.

\section{Discussion}

MRI is a powerful tool for the diagnosis of $\mathrm{TMD}^{3,9)}$, and can be used to observe structural changes in the TMJ, such as disc displacement, morphological disc change, fluid collection (effusion) in the articular space, change in the posterior band, and change in the bone marrow of the condylar head.

Effusion is depicted as a high signal intensity area on T2WI. Schellhas and Wilkes ${ }^{10)}$ reported that effusion was often observed in MR images of painful TMJs, and Larheim et al ${ }^{11)}$ suggested that moderate fluid was significantly associated with disc displacement. Studies have shown that the signal intensity of the posterior band increases in the following order: normal, posterior disc displacement with reduction, anterior disc displacement with reduction, and anterior disc displacement without reduction ${ }^{12}$. The signal intensity of the bone marrow in the condylar head is also used in the diagnosis of TMD. Larheim et al ${ }^{11)}$ reported that marked fluid effusion and abnormal signal intensity of the condylar marrow are observed in $10 \%$ of patients with TMD. In contrast, avascular necrosis is depicted as a low signal intensity area at the condylar marrow. Schellhas et al $^{13)}$ asserted that avascular necrosis, which is often associated with pre-existing internal derangement of the TMJ, is relatively common and results in clinically significant lesions of the mandibular condyle.

While the diagnostic assessments mentioned above were performed using only the MRI signal intensity, the articular disc and cortex of the condylar head are usually evaluated solely on their shape. Morphological changes of the disc are influenced by the type of displacement, and 
more serious deformations are associated with cases of anterior disc displacement without reduction $^{6}$. For the condylar cortex, MRI has been used to assess morphological changes, similar to computed tomography ${ }^{14)}$, however, in these studies, the signal intensities of the articular disc and condylar cortex were not taken into account. Conversely, other joints, such as the knee and hip, are assessed by both the shape and signal intensity of the articular cartilage ${ }^{15-17)}$.

There have been few studies investigating the signal intensity of the articular disc and condylar cortex. Kakimoto et al $^{8)}$ measured the $\mathrm{T} 2$ relaxation time of the articular disc, and concluded that the T2 relaxation time in patients with TMD was longer than in healthy volunteers. Accurate $\mathrm{T} 2$ relaxation time can be measured with a spin echo sequence. This is, however, too timeconsuming for use in the clinical setting. In clinical practice, a fast spin echo sequence is used to obtain T2WIs because of its short acquisition time. Bae et al ${ }^{18)}$ attempted to evaluate the articular disc quantitatively in terms of the $\mathrm{T} 1$ relaxation time, $\mathrm{T} 2$ relaxation time, and $\mathrm{T} 2 *$ relaxation time. They used a 3.0 tesla MRI unit and the ultrashort time-to-echo imaging technique. They found that the $\mathrm{T} 2 *$ value differed between asymptomatic and symptomatic volunteers. Although this is a very important result, at present, the 1.5 tesla MRI unit is the most common unit used clinically and the ultrashort time-to-echo technique remains difficult to use in the clinical setting.

We compared the attenuation patterns of the signal intensities of $\mathrm{CB}, \mathrm{AD}$, and $\mathrm{PD}$ in terms of TE. The results revealed that the signal intensities of $\mathrm{AD}$ and $\mathrm{PD}$ were higher than $\mathrm{CB}$ at TEs shorter than $20 \mathrm{msec}$. This indicates that the proton density of the articular disc is higher than the cortex. In other words, the articular disc contains more water than the cortex. At TEs longer than $20 \mathrm{msec}$, the signal intensities of $\mathrm{AD}$ and $\mathrm{PD}$ were lower than $\mathrm{CB}$, suggesting that the water in the disc has a shorter $\mathrm{T} 2$ relaxation time than in the cortex. These results likely reflect the differences in the composition and structure of cortical bone and the articular disc, namely, the amount of contained water and its form.

As for the components of normal bone, approximately $90 \%$ by weight is extracellular matrix, with $65 \%$ hydroxyapatite, $10 \%$ to $15 \%$ water, and approximately $25 \%$ composed of the organic components, type I collagen, and non-collagenous protein. A normal TMJ disc is composed of compact fibrous connective tissue, consisting mainly of type I collagenous fibers and fibroblasts. The vasculature is distributed at the periphery of the disc, particularly at the anterior band and the posterior band of the disc. We were unable to find any literature on the water content of human discs, and thus, could not verify whether the articular disc contains as much or more water than the cortex. The water content of the articular disc of cattle, however, is reported to be $66 \%$ to $80 \%$. It seems natural for the water content of the disc to be higher than that of the cortex. The shorter T2 relaxation time of the disc compared to the cortex may be caused by the form of the water, which is strongly bound to compact fibrous connective tissues ${ }^{19)}$.

The clinical PDWIs and T2WIs of patients in this study, which were obtained at TEs of $14 \mathrm{msec}$ and $85 \mathrm{msec}$, respectively, showed similar results to those obtained with the multi spinecho sequence, as shown in Fig. 5. These results provide important information on the measurement of signal intensities of the disc and cortex. Moreover, the signal intensities of AD, 
PD, and $\mathrm{CB}$ were significantly higher than that of the external auditory meatus (air) on both PDWIs and T2WIs. According to these results, it can be concluded that measurement of the signal intensities of the disc and cortex using a 1.5 tesla MRI unit with common imaging techniques is rational and valid.

Our results support the reliability of research on the relationship between disease states and the signal intensity of joint disks and cortical bone. In addition, these results suggest that further studies using signal intensity may provide new diagnostic information, especially for the early detection of inflammatory changes of the articular disc and cortex in TMD.

\section{Conflict of interest disclosure}

The authors declare no conflict of interest.

\section{References}

1) Helms CA, Richardson ML, Moon KL. et al. Nuclear magnetic resonance imaging of the temporomandibular joint: preliminary observations. J Craniomandibular Pract. 1984;2:219-224.

2) Roberts D, Pettigrew J, Ram C, et al. Radiologic techniques used to evaluate the temporomandibular joint; II. Computed tomography, three-dimensional imaging and nuclear magnetic resonance. Anesth Prog. 1984;31:241-256.

3) Brooks SL, Brand JW, Gibbs SJ, et al. Imaging of the temporomandibular joint: a position paper of the American Academy of Oral and Maxillofacial Radiology. Oral Surg Oral Med Oral Pathol Oral Radiol Endod. 1997;83:609-618.

4) Yatani H. New classification (2013) and its diagnostic criteria of temporomandibular disorders revised by the Japanese Society for Temporomandibular Joint. J Jpn Assoc Orthod. 2015;27:76-86. (in Japanese).

5) Basat SO, Surmeli M, Demirel O, et al. Assessment of the relationship between clinicophysiologic and magnetic resonance imaging findings of the temporomandibular disorder patients. J Craniofac Surg. 2016;27:1946-1950.

6) Amaral Rde O, Damasceno NN, de Souza LA, et al. Magnetic resonance images of patients with temporomandibular disorders: prevalence and correlation between disk morphology and displacement. Eur J Radiol. 2013;82:990-994.

7) Geiger D, Bae WC, Statum S, et al. Quantitative 3D ultrashort time-to-echo (UTE) MRI and micro-CT $(\mu \mathrm{CT}$ ) evaluation of the temporomandibular joint (TMJ) condylar morphology. Skeletal Radiol. 2014;43:19-25.

8) Kakimoto N, Shimamoto $\mathrm{H}$, Chindasombatjaroen J, et al. Comparison of the T2 relaxation time of the temporomandibular joint articular disk between patients with temporomandibular disorders and asymptomatic volunteers. AJNR Am J Neuroradiol. 2014;35:1412-1417.

9) Katzberg RW, Westesson PL. Magnetic resonance imaging. Cranio Clin Int. 1991;1:93-116.

10) Schellhas KP, Wilkes CH. Temporomandibular joint inflammation: comparison of MR fast scanning with T1- and T2-weighted imaging techniques. AJR Am J Roentgenol. 1989;153:93-98.

11) Larheim TA, Katzberg RW, Westesson PL, et al. MR evidence of temporomandibular joint fluid and condyle marrow alterations: occurrence in asymptomatic volunteers and symptomatic patients. Int J Oral Maxillofac Surg. 2001;30:113-117.

12) Orhan $\mathrm{K}$, Nishiyama $\mathrm{H}$, Tadashi $\mathrm{S}$, et al. Comparison of altered signal intensity, position, and morphology of the TMJ disc in MR images corrected for variations in surface coil sensitivity. Oral Surg Oral Med Oral Pathol Oral Radiol Endod. 2006;101:515-522.

13) Schellhas KP, Wilkes CH, Fritts HM, et al. MR of osteochondritis dissecans and avascular necrosis of the mandibular condyle. AJR Am J Roentgenol. 1989;152:551-560.

14) Karlo CA, Patcas R, Kau T, et al. MRI of the temporo-mandibular joint: which sequence is best suited to assess the cortical bone of the mandibular condyle? A cadaveric study using micro-CT as the standard of reference. Eur 
Radiol. 2012;22:1579-1585.

15) Link TM, Schwaiger BJ, Zhang AL. Regional articular cartilage abnormalities of the hip. AJR Am J Roentgenol. 2015:205:502-512.

16) Nishioka $H$, Nakamura $E$, Hirose J, et al. MRI $T 1$ rho and $T 2$ mapping for the assessment of articular cartilage changes in patients with medial knee osteoarthritis after hemicallotasis osteotomy. Bone Joint Res. 2016;5:294-300.

17) Van Rossom S, Smith $C R$, Zevenbergen $L$, et al. Knee cartilage thickness, $T 1$ rho and $T 2$ relaxation time are related to articular cartilage loading in healthy adults. PLoS One(Internet). 2017;12:e0170002.

18) Bae WC, Tafur M, Chang EY, et al. High-resolution morphologic and ultrashort time-to-echo quantitative magnetic resonance imaging of the temporomandibular joint. Skeletal Radiol. 2016;45:383-391.

19) Standring S. Gray's anatomy: the anatomical basis of clinical practice. 41st ed. [New York]: Elsevier; 2016.

[Received June 21, 2017 : Accepted August 15, 2017] 